\title{
Cutkosky rules for superstring field theory
}

\author{
Roji Pius $^{a}$ and Ashoke Sen ${ }^{b}$ \\ ${ }^{a}$ Perimeter Institute for Theoretical Physics, \\ Waterloo, ON N2L 2Y5, Canada \\ ${ }^{b}$ Harish-Chandra Research Institute, \\ Chhatnag Road, Jhusi, Allahabad 211019, India \\ E-mail: rpius@perimeterinstitute.ca, sen@mri.ernet.in
}

ERratum to: JHEP10(2016)024

ABSTRACT: Superstring field theory expresses the perturbative S-matrix of superstring theory as a sum of Feynman diagrams each of which is manifestly free from ultraviolet divergences. The interaction vertices fall off exponentially for large space-like external momenta making the ultraviolet finiteness property manifest, but blow up exponentially for large time-like external momenta making it impossible to take the integration contours for loop energies to lie along the real axis. This forces us to carry out the integrals over the loop energies by choosing appropriate contours in the complex plane whose ends go to infinity along the imaginary axis but which take complicated form in the interior navigating around the various poles of the propagators. We consider the general class of quantum field theories with this property and prove Cutkosky rules for the amplitudes to all orders in perturbation theory. Besides having applications to string field theory, these results also give an alternative derivation of Cutkosky rules in ordinary quantum field theories.

Keywords: String Field Theory, Superstrings and Heterotic Strings

ARXIV EPRINT: 1604.01783 


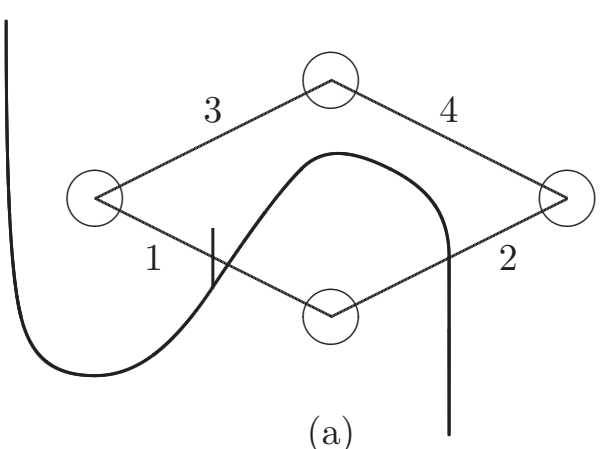

(a)

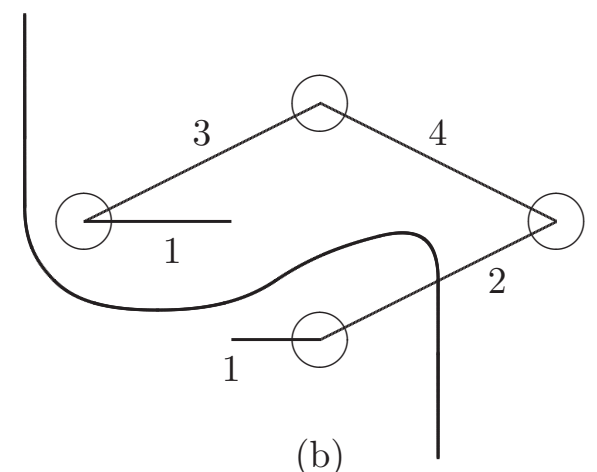

(b)

Figure 1. Figure (a) shows a cut reduced diagram in which a propagator $P_{1}$ is replaced by a cut propagator in the original diagram and the cut passes through the propagator $P_{1}$ in the reverse direction and the propagator $P_{2}$ in the correct direction. In our notation this will be labelled as $A_{\underline{1} 2}^{(1)}$. Figure (b) shows a more conventional depiction of the same diagram in which the cut propagator $P_{1}$ is depicted as one outgoing and one incoming particle carrying identical quantum numbers.

In the analysis described in section 5.2.3, while computing the anti-hermitian part of $A^{\left(i_{1} \cdots i_{s}\right)}$, we had included in the sum over cuts only the cuts of the original diagram. These were shown in figure 9 of [1]. However since in $A^{\left(i_{1} \cdots i_{n}\right)}$ the propagators $P_{i_{1}}, \cdots P_{i_{n}}$ are put on-shell, there are other possible cuts which, in the original diagram, will appear as if the cut passes through one or more of the propagators $P_{i_{1}}, \cdots P_{i_{n}}$ in the reverse direction. This has been shown in figure 1(a). While it may seem strange to include such cuts, as explained in figure 1(b), this is a regular cut if we regard each of the propagators $P_{i_{1}}, \cdots P_{i_{s}}$ as a pair of incoming and outgoing external particles carrying identical momentum. Putting a propagator on-shell effectively creates a gap in the propagator through which the cut can pass in either direction.

We shall now evaluate the contribution from these cut diagrams. As is clear from figure 1 if we have a cut passing through an on-shell propagator in the reverse order, there must be at least one other propagator in the set $\left\{P_{1}, \cdots P_{n}\right\}$ through which the cut passes through in the correct order - in figure 1(a) it is the propagator $P_{2}$. Without any loss of generality we can assume that among the on-shell propagators $P_{i_{1}}, \cdots P_{i_{s}}$, the propagators $P_{i_{1}}, \cdots P_{i_{m}}$ for $m \leq s$ are traversed by the cut in the reverse direction. We can express the contribution from these cut diagrams to $A-A^{*}$ as

$$
(-1)^{s} A_{\underline{i_{1} \cdots i_{m}} j_{1} \cdots j_{t}}^{\left(i_{1} \cdots i_{s}\right)},
$$

where $P_{j_{1}}, \cdots P_{j_{t}}$ are the propagators that are traversed by the cut in the right direction. The set $\left\{j_{1}, \cdots j_{t}\right\}$ may or may not have overlap with the set $\left\{i_{1}, \cdots i_{s}\right\}$. We now note that we can append to the set $\left\{i_{1} \cdots i_{s}\right\}$ in the superscript one or more members of the set $\left\{j_{1}, \cdots j_{t}\right\}$ that is not present there. This will not change the result since a cut passing through a cut propagator has no effect. Therefore we can begin with the term where $\left\{i_{1}, \cdots i_{s}\right\}$ has no overlap with $\left\{j_{1}, \cdots j_{t}\right\}$ and then add to it the result of appending one 
or more members of $\left\{j_{1}, \cdots j_{t}\right\}$. The total coefficient of such a term will be given by

$$
(-1)^{s}\left(1-t+\left(\begin{array}{l}
t \\
2
\end{array}\right)-\cdots+(-1)^{t}\right)=(-1)^{s}(1-1)^{t} .
$$

Since we have already argued that $t \geq 1$, we see that this contribution vanishes. Therefore there is no change in the final result, and eq. (5.31) of [1] gives the complete result for $A-A^{*}$.

Open Access. This article is distributed under the terms of the Creative Commons Attribution License (CC-BY 4.0), which permits any use, distribution and reproduction in any medium, provided the original author(s) and source are credited.

\section{References}

[1] R. Pius and A. Sen, Cutkosky Rules for Superstring Field Theory, JHEP 10 (2016) 024 [arXiv: 1604.01783] [INSPIRE]. 